Leny Alves Bomfim Trad'

Marcelo Eduardo Pfeiffer Castellanos'

Maria Clara da Silva

Guimarães"

Departamento de Saúde Coletiva I. Instituto de Saúde Coletiva - Universidade Federal da Bahia. Salvador, BA, Brasil

Programa de Pós-Graduação em Saúde Coletiva. Instituto de Saúde Coletiva. Universidade Federal da Bahia. Salvador, BA, Brasil

\footnotetext{
Correspondência | Correspondence:

Leny Alves Bomfim Trad

Instituto de Saúde Coletiva

Rua Basílio da Gama, $\mathrm{s} / \mathrm{n}^{\circ} 1^{\circ}$ andar - Canela

40110-040 Salvador, BA, Brasil

E-mail: trad@ufba.br
}

\title{
Acessibilidade à atenção básica a famílias negras em bairro popular de Salvador, Brasil
}

\section{Accessibility to primary health care by black families in a poor neighborhood of Salvador, Northeastern Brazil}

\section{RESUMO}

OBJETIVO: Analisar a acessibilidade de famílias negras de bairro popular aos serviços de atenção básica à saúde.

MÉTODOS: Estudo etnográfico, ancorado na antropologia de base interpretativa, realizado com 18 famílias selecionadas de um bairro popular de Salvador, BA, no período de dois anos. Os critérios de inclusão foram residência no bairro e autoclassificação como negros. A análise se baseou na antropologia interpretativa e considerou as categorias: autorreferência étnico-racial; experiências de discriminação nos serviços; percepção sobre acessibilidade na atenção básica; e barreiras de acessibilidade.

RESULTADOS E DISCUSSÃO: Identificaram-se os seguintes aspectos: a) identidade étnico-racial e saúde: percepção dos usuários de que as barreiras organizacionais e de acesso se devem a um amplo contexto social que produz cidadãos "de primeira e de segunda categorias", mais do que a um racismo institucional; b) acessibilidade no Sistema Único de Saúde (SUS): acesso problemático, permeado pela demora no atendimento, falta de compromisso dos profissionais de saúde, omissão dos gestores no controle e correção dessas situações; c) acessibilidade na atenção básica: visão sobre o contexto mais geral do SUS e apoio na descrição dos entrevistados sobre o acesso aos serviços de atenção básica.

CONCLUSÕES: Há barreiras de acessibilidade econômicas, organizacionais e culturais que se interpõem entre a oferta de serviços e o atendimento efetivo e oportuno das necessidades da população estudada.

DESCRITORES: Etnia e Saúde. Grupo com Ancestrais do Continente Africano. Acesso aos Serviços de Saúde. Atenção Primária à Saúde. Equidade no Acesso. Pesquisa Qualitativa. 


\begin{abstract}
OBJECTIVE: To analyse the accessibility of primary health care for black families from a poor neighbourhood.

METHODS: Ethnographic study with an interpretative anthropological approach, carried out with 18 families selected from a poor neighbourhood of Salvador, Northeastern Brazil, over a period of two years. Criteria for inclusion included being resident in the neighbourhood and classifying themselves as black. The analysis was based on interpretative anthropology and encompassed the following categories: ethnic and racial self-reference; experience of discrimination from public services; perception of accessibility to primary health care and barriers to accessibility.
\end{abstract}

RESULTS AND DISCUSSION: We identified the following aspects: a) ethnic and racial identity and health: the users' perception that organizational barriers and barriers to access are due to the wider social context which produces "first class" and "second class" citizens, rather than due to institutional racism; b) the accessibility of the Brazilian National Health System (Sistema Único de Saúde, SUS): difficult access, delays in being seen, lack of commitment on the part of health professionals, no management action taken to manage or improve these situations; c) accessibility of primary health care; overall vision of the context of the SUS and support in the interviewees descriptions of access to primary health care.

CONCLUSIONS: There are economic, organizational and cultural barriers to access which come between the service provided and effective care for the needs of the population of this study.

DESCRIPTORS: Ethnicity and Health. African Continental Ancestry Group. Health Services Accessibility. Primary Health Care. Equity in Access. Qualitative Research.

\section{INTRODUÇÃO}

Acesso e acessibilidade em saúde atraem cada vez mais a atenção dos pesquisadores da saúde coletiva, ao mesmo tempo em que constituem desafios contemporâneos da organização do Sistema Único de Saúde (SUS). ${ }^{8}$ Distintos usos e definições são identificados na operacionalização desses conceitos..$^{27}$ Acesso remete à disponibilidade dos serviços de saúde do ponto de vista da oferta. Acessibilidade trata da possibilidade concreta de aceder aos serviços ou recursos ofertados.

Travassos e Martins ${ }^{27}$ ponderam que

“acessibilidade (...) é mais abrangente do que a mera disponibilidade de recursos em um determinado momento e lugar. Refere-se às características dos serviços e dos recursos de saúde que facilitam ou limitam seu uso por potenciais usuários”.

O termo comporta dimensões socioeconômicas, geográficas, culturais e políticas do contexto social e assistencial que podem expressar-se como barreiras no atendimento das necessidades dos usuários (efetivos e/ou potenciais) do sistema de saúde. ${ }^{27} \mathrm{O}$ grau de acessibilidade pode ser mensurado pela relação entre a resistência apresentada por essas barreiras e a força de superação dos usuários ("poder de utilização”).

A garantia do acesso universal no SUS depende da identificação de eventuais barreiras vinculadas a características e necessidades da população. Sua heterogeneidade remete necessariamente a outro princípio básico do SUS: a equidade, que pode ser definida como

"ausência de diferenças sistemáticas potencialmente remediáveis em um ou mais aspectos da saúde em grupos ou subgrupos populacionais definidos socialmente, economicamente, demograficamente ou geograficamente” (Frenk ${ }^{9}$ ).

A equidade no acesso à saúde representa um dos maiores e mais complexos desafios contemporâneos em diversos países, requisitando estudos específicos e iniciativas na esfera das políticas públicas. ${ }^{28}$ Dentre os desafios para a pesquisa, destaca-se o refinamento de categorias capazes de capturar processos de produção e reprodução de 
“totalidades desvantajosas na exposição de certos indivíduos e grupos populacionais às ameaças à sua saúde e à construção de respostas para se defender delas” $\left(\right.$ Ayres $\left.^{3}\right)$.

Barreiras econômicas e étnico-raciais destacam-se entre os elementos que indicam a produção de iniquidades e contribuem para a vulnerabilização de diferentes grupos sociais. Classe social e pertença étnica são fatores importantes em processos de estigmatização social. Parker e Aggleton ${ }^{19}$ argumentam que esse processo guarda relação com estruturas de discriminação mais amplas expressas em determinadas tramas de relações entre cultura, poder e diferença (específicas a cada conjuntura social). A ênfase sobre a análise das relações entre os contextos de interação e as estruturas sociais mais amplas é defendida nas reflexões teóricas mais recentes sobre os processos de estigmatização. ${ }^{14,22}$

Estudos brasileiros identificam condições de vulnerabilidade, incluindo problemas de acessibilidade aos serviços de saúde, relacionadas a processos de estigmatização racial. Esses processos originam-se ao mesmo tempo em que afetam as condições de vida e o estado de saúde de determinados grupos, a exemplo da população negra. 4,7,15

A população negra brasileira apresenta um quadro de vulnerabilidade epidemiológica e social que inclui dificuldades de acesso aos serviços de saúde. ${ }^{16,18}$ Estudos epidemiológicos que adotaram o recorte racial na análise das taxas de mortalidade masculina ${ }^{5}$ e avaliação do acesso ao pré-natal e ao parto ${ }^{13}$ encontraram desvantagem da população negra, seguida da parda, quando comparadas com a branca.

A dupla “desvantagem” social (baixo capital econômico e simbólico) dessa população, produzida em um processo histórico complexo, desponta entre os aspectos determinantes desse quadro, o qual se aplica também à população indígena do País.

O presente estudo teve por objetivo analisar a acessibilidade de famílias negras de bairro popular aos serviços de atenção básica do SUS. Foram considerados os desafios e limites decorrentes das imprecisões em torno das categorias "população negra” ou "afrodescendente" no Brasil, bem como o caráter polêmico das análises que buscam associar traços raciais e indicadores de saúde. ${ }^{10,12}$ A complexidade inerente à análise das relações entre iniquidades e identidade étnica (e os conflitos sociais em torno desse fenômeno) é reconhecida em outros campos. ${ }^{20}$

\section{MÉTODOS}

Estudo etnográfico, parte de uma pesquisa mais ampla, ${ }^{a}$ que focalizou itinerários terapêuticos de famílias negras e redes de suporte sociossanitário. O estudo foi realizado com 18 famílias do bairro da Boca do Rio, na principal orla marítima urbana de Salvador, BA, num período de dois anos. O fato de o grupo de pesquisa desenvolver estudos na área desde 2005 foi decisivo na escolha do bairro.

Trata-se de um território com grandes contrastes urbanos, no qual antigas invasões, que ocupam a maior parte do bairro e concentram grande contingente populacional, fazem fronteira com condomínios de luxo. Esse cenário contrasta com o perfil dominante nos bairros populares da periferia da cidade, que mantêm maior distanciamento espacial das regiões mais abastadas. Por outro lado, segue a tendência dos bairros dessa categoria: apresenta predomínio de população negra.

A rede de saúde do bairro é insuficiente e desigualmente distribuída pelo território. $\mathrm{Na}$ área mais populosa e de menor poder aquisitivo existem duas unidades públicas de saúde (Atenção Básica e Centro de Atenção Psicossocial). Os moradores contam com serviço de pronto-atendimento localizado em setor mais distante, na fronteira com outro bairro. Existe pequena oferta de serviços privados (policlínica, consultórios dentários etc.) e de instituições não governamentais e comunitárias.

Foram realizadas observações participantes (centradas na rede de suporte sociossanitário do bairro) e entrevistas semiestruturadas com informantes-chave durante dois anos. Neste artigo, focalizaremos os dados dos estudos de caso com 18 famílias, gerados por entrevistas com informantes familiares.

Foram adotados os seguintes critérios de inclusão: 1) possuir residência no bairro; 2) autoclassificação de seus membros como negros, admitidos perfis raciais híbridos (classificação minoritária de seus membros como "moreno claro" ou "branco" - um caso); 3) ser SUS dependente. O número de famílias foi definido com base no princípio da saturação.

O termo "afrodescendente", adotado pelos pesquisadores, gerava reações que indicavam desconhecimento e/ou incompreensão na fase exploratória. Procurou-se identificar os termos/expressões que o grupo abordado/ entrevistado adotava para se referir à sua identidade "racial" ou cor.

A amostra (informantes familiares) apresentou o seguinte perfil sociodemográfico: mães, de 29 a 50 anos; com baixa escolaridade e exercício de ocupações informais de baixa remuneração. Foi recorrente a alusão a hipertensão arterial, diabetes, viroses infantis e agravos vinculados a episódios de violência no bairro como os principais problemas de saúde de suas famílias.

\footnotetext{
a "Itinerários Terapêuticos de Famílias afrodescendentes em um bairro popular de Salvador, Bahia".
} 
Realizaram-se em média duas entrevistas por família, totalizando 42, atendendo igualmente o princípio da saturação. As entrevistas foram conduzidas por uma dupla de pesquisadores, tiveram duração média de 1h10min, foram gravadas (com prévia autorização) e posteriormente transcritas.

Este artigo reúne resultados relativos às categorias: identidade étnico-racial, problemas de saúde, relação com a rede de serviços de saúde no bairro (particularmente, aspectos associados com acesso/acessibilidade), percepção e/ou vivências de racismo ou discriminação na esfera dos serviços.

Orientada pela antropologia interpretativa, a análise enfocou as inter-relações entre a experiência social e o contexto obtidas nas entrevistas (principal fonte) e observações. Identificaram-se os sentidos atribuídos pelos sujeitos às suas experiências e suas articulações com especificidades do contexto sociocultural (proximal e distal).

O Projeto foi aprovado pelo Comitê de Ética em Pesquisa do Instituto de Saúde Coletiva, Universidade Federal da Bahia (processo $n^{\circ}$ 024-09; 2009). Todos os participantes assinaram o Termo de Consentimento Livre e Esclarecido, conforme recomendações da Resolução nº 196/96 do Conselho Nacional de Saúde.

\section{RESULTADOS E DISCUSSÃO}

A cor negra foi expressa a partir de diferentes matizes: nego(a), negro(a), negão(ona), negrinho(a). Ressalta-se que “moreno(a)" e "moreninho(a)” apareceram com relativa frequência. Na produção do "racismo à brasileira”, o termo "moreno" pode ser considerado mais positivo que "negro" e mais específico do que "pardo". ${ }^{11}$

O preconceito racial foi tido como indissociável do preconceito de classe na percepção dos sujeitos sobre discriminação e racismo nos serviços de saúde. Embora a cidade de Salvador apresente população majoritariamente negra, fica patente a sobreposição entre raça e classe social ao se analisarem indicadores de vulnerabilidade social e epidemiológica. ${ }^{2,16}$

A classe social se sobressaiu à cor na questão sobre o aspecto mais determinante na produção da discriminação. Esses depoimentos encontravam na condição de "necessitado" a fonte principal das humilhações sofridas nos serviços:

“Não por causa de cor. Eles humilham muito, qualquer tipo de pessoa que chega lá precisando” (Celeste).

Muitos entrevistados afirmaram que o fato de o SUS ser responsável basicamente por ofertar assistência aos "pobres" incide diretamente sobre a qualidade dos serviços prestados, a infraestrutura, condições de acesso etc. Essa foi a referência mais contundente às relações entre classe social e discriminação nos serviços (iniquidade sanitária).

Para aqueles que atribuíam dada experiência de discriminação à sua condição de pobreza, o fato de ser negro potencializaria situações discriminatórias. A presunção da pobreza e da existência de déficits educacionais e culturais entre os negros seria propulsora da estigmatização desse grupo social, conforme mostra uma das entrevistadas.

"Pelo fato de ser preta? Não. Eu acho que já me senti discriminada pelo fato de ser pobre. Porque os outros olham você assim. Às vezes, juntam as duas coisas. As pessoas discriminam os negros achando que o negro é pobre. Que não tem estudo, não tem cultura, não tem nada” (Flávia).

A análise das imbricações entre atributos que acarretam discriminação e estigmatização poderá mostrar o impacto desses processos na vida das pessoas afetadas, na medida em que incidem sobre o acesso a recursos e posições sociais. ${ }^{6,14}$

Foi referido um episódio concreto de discriminação ou racismo na relação com os serviços públicos de saúde.

“A médica pega assim [gesto com dois dedos mal tocando na pele], nem pega na criança (...) bole com nojo das crianças, por mais que ela [a criança] esteja tomada banho, bonitinha, cheirosinha. É assim mermo, assim mermo, porque é morena” (Clarissa).

A narrativa sugere que a discriminação racial pode ser interpretada no gesto sutil (mesmo dúbio), ganhando posteriormente acento nas palavras que legitimamente retomam e explicitam tal gesto. O uso do termo "morena” para se referir à filha de cor negra corrobora as observações de Guimarães. ${ }^{11}$

Os processos de discriminação nos serviços de saúde, cujas repercussões materiais e simbólicas são significativas, devem ser reconhecidos como elementos centrais quando se pensa efetividade e equidade no SUS. Entretanto, não são alvo destacado de pesquisa. ${ }^{26} \mathrm{O}$ fato de não terem sido encontrados registros significativos de ocorrência de episódios de discriminação nos serviços de saúde na presente pesquisa não invalida o argumento em defesa de maior investimento na investigação sobre o tema.

À medida que adentrávamos na discussão sobre a relação cotidiana com a rede de serviços, sobretudo com a atenção básica, iam perdendo força as remissões a elementos de caráter étnico-racial. A condição de pobreza da população afetada pelos problemas narrados emerge como fator que explicaria o “descaso das autoridades” com relação a ela.

As impressões e experiências dos informantes conformam um corolário de queixas sobre os serviços semelhante 
àquele de estudos que avaliaram a atenção básica em outros municípios da Bahia ou do Nordeste (contemplando áreas nas quais a população predominante era parda ou branca). ${ }^{23-25}$

A percepção dos sujeitos sobre os serviços de atenção básica reitera estereótipos associados a esse nível da atenção: baixa densidade tecnológica, pouca resolutividade, predomínio da oferta de ações paliativas, realizadas por profissionais não especializados e caracterizadas pela simplicidade/escassez de recursos terapêuticos visão que vem acompanhada da demanda pela criação e expansão de ambulatório de especialidades na rede básica.

“No posto de saúde, eles só dão um paliativo (...) é pra primeiros socorros, eles não dão tratamento (...) é um remédio pra dor, é um anti-inflamatório, antibiótico (...) não tem áreas especificas” (Clara).

"Principalmente, saúde bucal (...) O posto dá assim um suporte de extrair um dente, uma obturaçãozinha, uma limpeza” (Roberta).

Aspectos que remetem a deficiências organizacionais ou insatisfação com a lógica de organização foram encontrados na análise de eventuais barreiras ou obstáculos que se interpõem entre os usuários e os serviços. ${ }^{9}$ Foram referidos ainda limites associados ao comportamento dos usuários, como a prática de marcar consulta para o mesmo procedimento em diferentes unidades.

O elevado tempo de espera na unidade ou no agendamento de consultas-procedimentos despontou entre os alvos de insatisfação das famílias: "Nunca é atendido na hora certa” (Roberta); "dentista... um ano” (Clara); "ginecologista daqui a dois meses" (Jussara). O horário restrito reservado a consultas médicas ou odontológicas, comprovado pela observação, foi outro alvo de queixa. Alguns informantes ressentiam-se da falta de interferência dos gestores ("prefeitos", "ministro") no controle do cumprimento da carga horária dos profissionais.

O atendimento a pessoas de outros bairros ou cidades foi apontado como responsável por agravar esse problema:

"Vem gente até do interior (...) quando chega nossa vez, fica sem consulta" (Clara).

O pertencimento ao território é reconhecido, portanto, como critério organizacional legítimo para definir o acesso aos serviços locais. Foi precisamente esse critério, considerado estratégico para a oferta organizada de serviços, que justificou a incorporação do cadastramento da população local no Programa de Saúde da Família.

O critério de priorização de grupos de risco foi polemizado por alguns entrevistados, que argumentaram em favor da "ordem de chegada" como um critério justo para orientar $\mathrm{o}$ atendimento.
"Só idoso tem prioridade, e deficiente. Às vezes, dá raiva, você tá chegando duas horas da manhã pra pegar uma ficha pro médico. Aí, quando dá sete horas, (...) chegam vinte idosos. Eles têm que ter a prioridade deles, têm que ter um médico pra eles. Não passar na frente da gente. Às vezes, eles pegam todas as fichas e a gente volta pra casa sem as fichas” (Flávia).

Destaca-se, acima, a crítica ao tratamento diferenciado concedido aos idosos. Considerando-se a tendência demográfica de expansão desse grupo etário, o dado merece atenção especial dos gestores de saúde. Embora o critério de priorização atenda a um requisito legal e possa ser considerado equitativo, é quase sempre fonte de questionamento. Pesquisas realizadas em unidades de Saúde da Família encontraram resistência dos usuários em relação à adoção de critérios de prioridade na distribuição de fichas para consultas..$^{24,25}$

Certas normas para o atendimento nos serviços de saúde foram consideradas rígidas e entendidas como "burocracia”. Reitera-se o impasse entre a lógica da oferta, definida pelos serviços, e as expectativas dos usuários, que remetem a necessidades diversas. ${ }^{1,28}$

A interveniência de contatos pessoais, que depende das vinculações da rede social da família com os serviços para garantir o atendimento, foi outro ponto de debate. Se para uns essa é a única maneira de conseguir minimizar as dificuldades de acesso, para outros configura uma situação de favorecimento:

"tem a prioridade dos amigos, dos parentes, que elas botam na frente. Se botar na minha, eu armo um barraco” (Flávia).

Alguns entrevistados referiram realizar agendamento em diferentes unidades para driblar a demora na marcação de consultas. Essa conduta retroalimenta os déficits na organização da oferta de serviços. A busca da rede privada foi apontada como uma alternativa acionada quando não é possível esperar pelo atendimento público.

Um dos entrevistados lamentou o fato de não poder contar mais com o plano de saúde em consequência da perda do emprego: "quando eu trabalhava (...) eu ia, é claro, no particular. Eu tinha um convênio" (Mariana). Houve tendência em idealizar a organização e qualidade dos serviços na rede privada de saúde.

A iniquidade racial e socioeconômica acentuam-se no setor privado. $\mathrm{O}$ acesso aos planos de saúde no Brasil é fortemente concentrado nos indivíduos com maior renda familiar, de cor branca e com alto nível de escolaridade. ${ }^{21}$ Paradoxalmente, esse panorama favorece a percepção, especialmente entre aqueles que não possuem planos de saúde, de que os serviços privados têm melhor qualidade do que os públicos. ${ }^{8}$ 
A alta rotatividade dos profissionais foi outro alvo de críticas. Houve descontentamento com esse fato, principalmente quando implicava a quebra de vínculo com um profissional bem avaliado: "De vez em quando, tem um pediatra bom no posto, você tá acostumado a levar uma criança lá. Daqui a pouco, quando chega, cadê?" (Celeste).

A estabilidade da equipe foi apontada como um fator que favorecia o acolhimento no serviço e a confiança nos profissionais. Além disso, o fato de se sentir reconhecido ("lembrado") pelos profissionais de saúde foi valorizado pelas famílias, reiterando estudos anteriores. ${ }^{24,25}$

“O povo que trabalha lá é uma bênção, fora a recepcionista. O dia que eu não vou, elas (...) ficam procurando saber uma da outra, das pessoas que vão pra curativo. 'Por que Clara não veio hoje?', (Clara).

Diferenças na postura de profissionais foram atribuídas a características pessoais (índole, educação familiar), culturais ou, ainda que em menor grau, à insatisfação com as condições de trabalho (sobrecarga, baixos salários e outros):

"tem aqueles funcionários que são educados, são preparados pra trabalhar ali. E tem aqueles que (...) têm o mau humor" (Roberta).

Observou-se uma situação paradoxal quanto à filiação religiosa. As redes religiosas figuraram como opção recorrente nos itinerários terapêuticos das famílias. Por outro lado, a filiação religiosa e/ou adesão aos respectivos preceitos foi um obstáculo na relação com os serviços de saúde (SUS ou organizações não governamentais) em alguns casos. Emergem a intolerância religiosa e os preconceitos perante as crenças alheias. Uma das informantes justificou sua recusa em recorrer ao centro de saúde, mantido por uma instituição espírita local, nos seguintes termos:

"Eu não frequento [o serviço] porque eu sou testemunha de Jeová. Os irmãos (...) não proíbem (...) se eu leio minha bíblia, sei que diz que é proibido (...) convivência com pessoas espíritas (...) Eles devem levar a criança pra, sei lá, hipnotizar, sei lá, como é que eles fazem, como é o tratamento deles" (Mariana).

Alguns agentes comunitários de saúde (ACS), adeptos da religião evangélica, recusavam-se a visitar casas do bairro onde funcionam terreiros de candomblé (foram registrados 13 terreiros na Boca do Rio), o que preocupava a equipe gestora local.

Uma das informantes (“mãe de santo”) ressaltou o papel histórico do candomblé no acolhimento da população negra e na defesa de seus valores culturais, o que para ela contribui para o preconceito contra o "povo de santo”. Raça e religião somam-se na produção da discriminação.

A questão da pertença étnico-racial foi raramente acionada quanto à acessibilidade aos serviços. Além do aspecto referido acima, foi mencionado o “descaso” ou negligência em relação às doenças falciformes, reconhecidas como um agravo prevalente entre negros no município de Salvador.

\section{CONSIDERAÇÕES FINAIS}

Os sujeitos associaram manifestações de estigma e discriminação social (observadas ou vivenciadas) mais à condição de pobreza do que à cor da pele. Prevaleceu a visão de que os processos de discriminação social no Brasil, que se expressam também na saúde, fazem parte de uma conjuntura social mais ampla que produz cidadãos de primeira e de segunda categorias ("pessoas de sandália”). Configura-se, assim, um arranjo complexo entre os capitais econômicos e simbólicos.

Por outro lado, parte dos entrevistados encontra na combinação “ser negro e pobre” um elemento que potencializa as dificuldades na relação com os serviços de saúde. A ausência de estratégias que permitissem comparar a percepção de negros e não negros no mesmo território - o que poderia oferecer pistas acerca da influência do quesito cor de pele nos resultados encontrados - pode ser apontada como um dos limites do estudo.

Estudos que permitam precisar o peso específico de marcadores étnico-raciais e de classe social na acessibilidade nos serviços de saúde, bem como na eventual ocorrência de discriminação neste contexto, devem ser realizados. Será conveniente que tais pesquisas contemplem regiões do País com diferentes perfis étnico-raciais e comparem condições de acessibilidade em saúde entre grupos populacionais com características diversas em termos de cor e classe social.

\section{REFERÊNCIAS}

1. Acúrcio FA, Guimaraes MDC. Acessibilidade de indivíduos infectados pelo HIV aos serviços de saúde: uma revisão de literatura. Cad Saude Publica. 1996;12(2):233-42. DOI:10.1590/S0102-311X1996000200012

2. Araujo EM, Costa MCN, Oliveira NF, Santana, FS, Barreto ML, Hogan V, et al. Distribuição espacial da

mortalidade por homicídio e desigualdades sociais segundo a raça/cor em um espaço intra-urbano no Brasil. Rev Bras Epidemiol. 2010;13(4):549-60. DOI:10.1590/S1415-790X2010000400001

3. Ayres JRCM. Raça como conceito emancipador e vulnerabilidade como norte para políticas de equidade 
em saúde. Cad Saude Publica. 2007;23(3):497-523. DOI:10.1590/S0102-311X2007000300009

4. Barata RB, Ribeiro MCSA, Cassanti AC. Social vulnerability and health status: a household survey in the central area of a Brazilian metropolis. Cad Saude Publica. 2011;27(Suppl2):164-75. DOI:10.1590/S0102-311X2011001400005

5. Batista LE. Masculinidade, raça/cor e saúde. Cienc Saude Coletiva. 2005;10(1):71-80. DOI:10.1590/S1413-81232005000100013

6. Castro A, Farmer P. Understanding and addressing aidsrelated stigma: from anthropological theory to clinical practice in Haiti. Am J Public Health. 2005;95(1):53-9. DOI:10.2105/AJPH.2003.028563

7. Chor D, Lima CRA. Aspectos epidemiológicos das desigualdades raciais em saúde no Brasil. Cad Saude Publica. 2005;21(5):1586-94. DOI:10.1590/S0102-311X2005000500033

8. Conill EM, Pires D, Sisson MS, Oliveira MC, Boing AF, Fertonani HP. O mix público-privado na utilização de serviços de saúde: um estudo dos itinerários terapêuticos de beneficiários do segmento de saúde suplementar brasileiro. Cienc Saude Coletiva. 2008;13(5):1501-10. DOI:10.1590/S1413-81232008000500015

9. Frenk J. El concepto y medición de la accesibilidad. Salud Publica Mex. 1985;27(5):438-53.

10. Fry PH, Monteiro S, Maio MC, Bastos Fl, Santos RV. AIDS tem cor ou raça? Interpretação de dados e formulação de políticas de saúde no Brasil. Cad Saude Publica. 2007;23(3):497-507. DOI:10.1590/S0102-311X2007000300002

11. Guimarães AS. Racismo e Anti-Racismo no Brasil. 2 ed. São Paulo: Editora 34; 2005.

12. Laguardia J. O Uso da Variável "Raça" na pesquisa em saúde. Physis. 2004;14(2):197-234. DOI:10.1590/S0103-73312004000200003

13. Leal MC, Gama SGN, Cunha CB. Desigualdade raciais no pré-natal e no parto. Rev Saude Publica. 2005;39(1):100-7. DOI:10.1590/S0034-89102005000100013

14. Link BG, Phela JoC. Stigma and its public health implications. Lancet. 2006;367(9509):528-9. DOI:10.1016/S0140-6736(06)68184-1

15. Lopes F, Buchalla CM, Ayres JRCM. Mulheres negras e não-negras e vulnerabilidade ao HIV/Aids no estado de São Paulo, Brasil. Rev Saude Publica. 2007;41(2):3946. DOI:10.1590/S0034-89102007000900008
16. Lopes F. Para além da barreira dos números: desigualdades raciais e saúde. Cad Saude Publica. 2005;21(5):1595-601. DOI:10.1590/S0102-311X2005000500034

17. Macinko JA, Starfield B. Annotated bibliography on equity in health, 1980-2001. Int I Equity Health. 2002;1(1):1980-2001. DOI:10.1186/1475-9276-1-1

18. Oliveira F. Ser negro no Brasil: alcances e limites. Estud Av. 2004;18(50):57-60 DOI:10.1590/S0103-40142004000100006

19. Parker R, Aggleton P. Estigma, discriminação e AIDS. Rio de Janeiro: Associação Brasileira Interdisciplinar de AIDS; 2001.

20. Pellow D. Social inequalities and environmental conflict. Horizontes Antropol. 2006;12(25):15-29. DOI:10.1590/S0104-71832006000100002

21. Pinto L. Planos privados de assistência à saúde: cobertura populacional no Brasil Cienc Saude Coletiva. 2004;9(1):85-98. DOI:10.1590/S1413-81232004000100009

22. Scambler G. Health-related stigma. Sociol Health Illness. 2009;31(3):441-55. DOI:10.1111/j.1467-9566.2009.01161.x

23. Souza ECF, Vilar RLA, Rocha NSPD, Uchoa AC, Rocha PM. Acesso e acolhimento na atenção básica: uma análise da percepção dos usuários e profissionais de saúde. Cad Saude Publica. 2008;24(Supl 1):100-10. DOI:10.1590/S0102-311X2008001300015

24. Trad LAB, Esperidião MA. Sentidos e práticas da humanização no PSF: a visão de usuários em seis municípios do Nordeste. Physis. 2010;20(4):1099-117. DOI:10.1590/S0103-73312010000400003

25. Trad LAB, Bastos ACS, Santana EM, Nunes MO. Estudo etnográfico de satisfação de usuário do Programa de Saúde da Família (PSF) na Bahia. Cienc Saude Coletiva. 2002;7(3):581-9. DOI:10.1590/S1413-81232002000300015

26. Travassos C, Bahia L. Qual é a agenda para o combate à discriminação no SUS? Cad Saude Publica. 2011;27(2):204-5. DOI:10.1590/S0102-311X2011000200001

27. Travassos C, Martins M. Uma revisão sobre os conceitos de acesso e utilização de serviços de saúde. Cad Saude Publica. 2004;20(Suppl2):190-8. DOI:10.1590/S0102-311X2004000800014

28. Van Doorslaer E, Masseria C, Koolman X. Inequalities in access to medical care by income in developed countries. CMAJ. 2006;174(2):177-83. DOI:10.1503/cmaj.050584 